\title{
Tetrabutylammonium Bromide-Promoted Metal-Free, Efficient, Rapid, and Scalable Synthesis of N-Aryl Amines
}

\author{
Chad R. Johnson, ${ }^{\dagger}$ Mohd Imran Ansari, ${ }^{\dagger}$ and Andrew Coop*๑
}

Department of Pharmaceutical Sciences, University of Maryland School of Pharmacy, 20 N. Pine Street, Baltimore, Maryland 21201, United States

\section{Supporting Information}

ABSTRACT: A rapid, transition metal-free, high-yielding, tetrabutylammonium bromide-promoted method of $\mathrm{N}$-arylation is reported within. The optimized conditions tolerated a wide range of secondary amines and was equally effective with bromo- and chlorobenzene-including substituted aryl halides. The developed method is found to be effective for $\mathrm{N}$-arylation when compared to earlier methods which involve harsh conditions, transition metals, lack of scalability, and long reaction times. Our method utilizes conventional heating only; it is readily scalable; and the products are facile to purify.

\section{INTRODUCTION}

Recent advancements in $\mathrm{N}$-aryl bond formation have motivated medicinal chemists to synthesize biologically important $\mathrm{N}$-arylated compounds, which earlier proved difficult to prepare. ${ }^{1}$ Because of the abundance of natural products, drugs of pharmaceutical importance, pesticides, and color pigments containing $\mathrm{N}$-arylated amines, efficient methods have been developed, including Buchwald-Hartwig and Ullmann couplings, among others. ${ }^{2}$ Copper or palladium metals, with or without other ligands, are generally required for the synthesis of $\mathrm{C}-\mathrm{N}$ bonds in these well-known reactions. ${ }^{2}$

Though these reactions are widely used for the synthesis of aryl amines, some suffer several drawbacks as they require the use of a metal, harsh reaction conditions, and costly reagents. Because contamination of the product with the transition metal is of concern, much effort has been devoted toward the development of metal-free $\mathrm{N}$-arylation reactions. ${ }^{3}$ Recently, Huang et al. reported the metal-free synthesis of $\mathrm{N}$-aryl tertiary amines in which they reacted methyl and ethyl-derivatized tertiary amines with various aryl halides with excess potassium tert-butoxide to obtain the $\mathrm{N}$-arylated product. ${ }^{4}$ Fang and coworkers also used aliphatic tertiary amines for the synthesis of arylated amines by using potassium hydroxide as a base. ${ }^{5}$ Shi et al. developed a rapid microwave-assisted synthesis of secondary and tertiary aryl amines in dimethyl sulfoxide (DMSO) and excess potassium tert-butoxide in good yields. ${ }^{6}$ Although the above methods were effective in generating the $\mathrm{N}$-arylated products, the method of Fang et al. required $24 \mathrm{~h}$ to obtain moderate yields. Shi's method requires the use of a microwave reactor, making scalability a challenge. In an attempt to identify a simple, efficient, and scalable method for the synthesis of $\mathrm{N}$ aryl amines, we report rapid, metal-free tetrabutylammonium bromide (TBAB)-promoted arylation of secondary amines using bromo- and chlorobenzene.

\section{RESULTS AND DISCUSSION}

Bromobenzene $(0.20 \mathrm{~mL}, 2 \mathrm{mmol})$ and morpholine $(0.26 \mathrm{~mL}$, $3 \mathrm{mmol}$ ) were chosen as substrates for reaction optimization (Scheme 1) with potassium tertiary butoxide $\left(\mathrm{KO}^{t} \mathrm{Bu}\right)$ as the

Scheme 1. Optimized Conditions for the Reaction
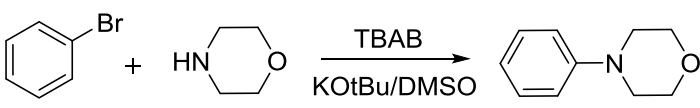

$130^{\circ} \mathrm{C}$

base in DMSO $(2 \mathrm{~mL})$. We first studied the protocol of Shi et al. for large-scale synthesis by simply heating for $12 \mathrm{~h}$ with no microwave (Scheme 1). ${ }^{6}$ The method was successful in generating the $\mathrm{N}$-arylated product, but only in $65 \%$ yield. Prolonged reaction time (greater than $12 \mathrm{~h}$ ), increasing the temperature, or increasing the amount of base failed to increase the yields. After reviewing the literature, we found that TBAB has been successfully used in several carbonheteroatom and carbon-carbon bond formation reactions, halogenation, and oxidation reactions. Johnson et al. utilized TBAB to efficiently alkylate sulfoximines; ${ }^{7}$ Wang et al. employed $\mathrm{TBAB}$ to rapidly alkylate acridone in a high yield; ${ }^{8}$ Majumdar et al. reported a TBAB-promoted S-alkylation of 4mercapto-6-methyl-2-pyrone with allyl and propargyl halides under mild conditions with moderate to high yields; ${ }^{9}$ and Lebel et al. reported the synthesis of a complex polydentate phosphane ligand by utilizing TBAB-catalyzed P-alkylation of phosphane borane in excellent yields. ${ }^{10}$

Encouraged by the successful alkylations noted above under phase-transfer conditions, we also searched the literature for examples of $\mathrm{TBAB}$ being used under nonphase transfer

Received: June 22, 2018

Accepted: August 30, 2018

Published: September 10, 2018 
conditions. $\mathrm{Li}$ et al. and Tang et al. noted that TBAB could serve as an activator/additive to promote both substitution and cross-coupling reactions, respectively. ${ }^{11}$ On the basis of these findings, we attempted to utilize $\mathrm{TBAB}$ under nonphase transfer conditions. We were encouraged to see that complete consumption of the starting material occurred after $30 \mathrm{~min}$ with an isolated yield of $85 \%$ when $50 \mathrm{~mol} \%(161 \mathrm{mg})$ TBAB was used. Hoping to further optimize the reaction conditions, we increased the loading of $\mathrm{TBAB}$ but were met with decreased yields. TBAB loading was then lowered systematically until it was found that $5 \mathrm{~mol} \%$ was optimal (Figure 1).

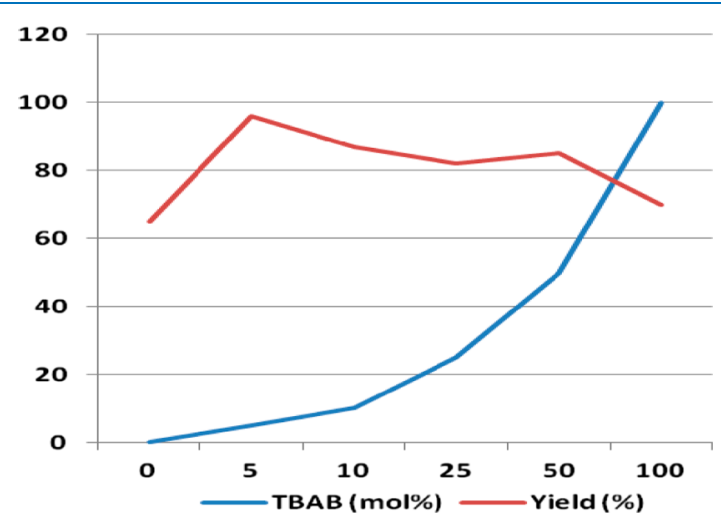

Figure 1. TBAB optimization graph.

Using these conditions, $N$-phenylmorpholine (Table 1, entry 9) was obtained in $95 \%$ yield in only $8 \mathrm{~min}$. When the reaction was conducted at $100{ }^{\circ} \mathrm{C}$, the reaction furnished the $\mathrm{N}$-arylated product in excellent yield but required greater than double the time interval for completion. Below $100{ }^{\circ} \mathrm{C}$, the reaction was sluggish and required several hours to complete.

We then screened different solvents such as tetrahydrofuran, EtOH, dimethylformamide, and $N$-methylpyrrolidone (NMP) to assess if further optimization was possible. NMP proved to be the only other suitable solvent for our reaction conditions, but produced lower yield than that obtained from DMSO. After the solvent, time, and TBAB loading conditions were established, we carried out multiple $\mathrm{N}$-arylation reactions with several different secondary amines. This method was equally effective with all secondary amines tested, and the products were furnished in good to excellent yields (Table 1). Bromobenzenes substituted with electron-donating or -withdrawing groups were also found to be as effective as unsubstituted bromobenzene, yielding the mixture of isomers in their expected ratios ${ }^{5,6}$ (Table 1). To test the scalability, three secondary amines, dibutyl amine, piperidine, and morpholine, were selected for reaction with bromobenzene (Table 1 entries 24, 25, and 26) on a $3 \mathrm{~g}$ scale. All reactions were completed within $20 \mathrm{~min}$ in excellent yield (Table 1).

To further investigate the scope and limitation of the reaction, chlorobenzene was also chosen as a substrate. The reaction was completed in equal time and a comparable yield to furnish 1-phenylmorpholine. N-Arylated product with these optimized conditions showed either better or comparable performance with earlier reported reactions in which metal catalysts were used. ${ }^{1-3,11}$ On further application to various alkyl amines, we found that that the $\mathrm{N}$-arylation of indole (Table 1, entry 10, yield 76\%), 1,2,3,4-tetrahydroquinoline (Table 1 , entry 11 , yield $86 \%$ ), tetrahydroisoquinoline (Table 1 , entry 12 , yield $91 \%$ ), even the sterically crowded dicyclohexylamine (Table 1 , entry 6 , yield $82 \%$ ), and diphenylamine (Table 1, entry 14, yield $79 \%$ ) all proceeded in good to excellent yields in short reaction times.

It is accepted that the reaction proceeds mechanistically through the generation of a benzyne intermediate (slow) followed by its rapid quenching via the desired nucleophile (fast), as explained by the mixture of isomers obtained from 1bromo-4-fluorobenzene, 4-bromotoluene, and 4-bromoanisole with morpholine (entries 21, 22, and 23). ${ }^{5,6}$ The reactions were completed within $15 \mathrm{~min}$, and isomers of the products were formed in a 1:1 ratio, as indicated by nuclear magnetic resonance (NMR) spectroscopy (see the Supporting Information).

\section{CONCLUSIONS}

$\mathrm{C}-\mathrm{N}$-aryl bond formation is one of the most challenging tasks in medicinal chemistry, with scalability and metal contamination being major concerns with the current approaches. Herein, we have detailed a scalable, high-yielding, and efficient TBAB-promoted $\mathrm{N}$-arylation of several secondary amines. Future studies will focus on determining the mechanism through which $\mathrm{TBAB}$ promotes this reaction.

\section{EXPERIMENTAL SECTION}

General Information. Reagents and solvents were of ACS grade and purchased from Sigma-Aldrich or Alfa Aesar. Inert atmosphere $\left(\mathrm{N}_{2}\right.$ or argon) is not required. Anhydrous solvents were used as provided without further purification. Reactions were monitored by thin-layer chromatography (TLC), visualizing with an ultraviolet lamp and $\mathrm{I}_{2}$. Flash column chromatography was performed on RediSep Rf Gold columns with a Whatman Purisil 60A silica gel (230-400 mesh) loading column on a Teledyne Isco CombiFlash Rf. ${ }^{1} \mathrm{H}$ NMR spectra were recorded on a Varian INOVA $400 \mathrm{MHz}$ NMR spectrometer at $25{ }^{\circ} \mathrm{C}$. All synthesized compounds were characterized by ${ }^{1} \mathrm{H}$ NMR and electrospray ionization (ESI) mass spectrometry analysis (in the case of 1-bromo-4fluorobenzene). Chemical shifts are reported in parts per million. The residual solvent peak was used as the internal reference: $\mathrm{CDCl}_{3}$. Mass spectra were obtained on an ESI timeof-flight mass spectrometer (Bruker amaZon X).

General Procedure for the Synthesis of Tertiary Amines. To a dry round-bottom flask (or pressure vial for low boiling amines) was added bromobenzene/chlorobenzene $(2.0 \mathrm{mmol})$ and secondary amine $(3.0 \mathrm{mmol})$ in $3 \mathrm{~mL}$ of DMSO, followed by TBAB $(32.0 \mathrm{mg}, 5 \mathrm{~mol} \%)$ and 1.5 equiv potassium tert-butoxide $(337.0 \mathrm{mg}, 3.0 \mathrm{mmol})$. The reaction was heated at $130{ }^{\circ} \mathrm{C}$ and monitored by TLC at frequent time intervals. The reaction mixture was allowed to cool, diluted with water, and extracted with ethyl acetate $(4 \times)$. The organic layers were combined, washed with brine, dried over $\mathrm{MgSO}_{4}$, filtered, and concentrated in vacuo. The crude reaction mixture was purified by column chromatography (0-20\% ethyl acetate/hexanes) to provide the desired tertiary amines. All resulting products were verified by NMR (directly compared with the result from previously published literature).

$N, N$-Diethylaniline (1). $263 \mathrm{mg}, 88 \%$ yield, clear to light yellow liquid; ${ }^{1} \mathrm{H} \mathrm{NMR}\left(\mathrm{CDCl}_{3}, 400 \mathrm{MHz}, \mathrm{ppm}\right): \delta 7.24-7.18$ $(\mathrm{m}, 2 \mathrm{H}), 6.70-6.63(\mathrm{~m}, 2 \mathrm{H}), 3.36-3.32(\mathrm{~m}, 6 \mathrm{H}), 1.18-1.13$ $(\mathrm{m}, 6 \mathrm{H}){ }^{6}$

$\mathrm{N}, \mathrm{N}$-Dipropylaniline (2). $301 \mathrm{mg}, 85 \%$ yield, light brown liquid; ${ }^{1} \mathrm{H}$ NMR $\left(\mathrm{CDCl}_{3}, 400 \mathrm{MHz}, \mathrm{ppm}\right): \delta 7.20-7.16(\mathrm{~m}$, 
Table 1. TBAB-Promoted Reaction of Bromo/Chlorobenzene with Secondary Amines ${ }^{a}$

\begin{tabular}{|c|c|c|c|c|c|c|}
\hline \multirow[b]{3}{*}{ Entry } & \multirow{2}{*}{\multicolumn{2}{|c|}{$A r X+H N^{R_{1}}$}} & \multirow{2}{*}{$\underset{\substack{\mathrm{KOtBu}, \mathrm{DMSO} \\
130^{\circ} \mathrm{C}}}{5 \mathrm{~mol} \% \mathrm{TBAB}} \longrightarrow \mathrm{Ar}-\mathrm{N}_{\mathrm{R}_{2}}^{\mathrm{R}_{1}}$} & \multirow[b]{3}{*}{ Time $^{\text {b }}$} & \multirow[b]{3}{*}{ Yield $^{\mathrm{c}}$} & \multirow[b]{3}{*}{ Reference } \\
\hline & & & & & & \\
\hline & ArX & Amine & Product & & & \\
\hline 1 & $\mathrm{PhBr}$ & $\left(\mathrm{CH}_{3} \mathrm{CH}_{2}\right)_{2} \mathrm{NH}$ & $\left(\mathrm{CH}_{3} \mathrm{CH}_{2}\right)_{2} \mathrm{NPh}$ & 12 & 88 & 6 \\
\hline 2 & $\mathrm{PhBr}$ & $\left(\mathrm{CH}_{3} \mathrm{CH}_{2} \mathrm{CH}_{2}\right)_{2} \mathrm{NH}$ & $\left(\mathrm{CH}_{3} \mathrm{CH}_{2} \mathrm{CH}_{2}\right)_{2} \mathrm{NPh}$ & 12 & 85 & 5 \\
\hline 3 & $\mathrm{PhBr}$ & $(\mathrm{i}-\mathrm{Pr})_{2} \mathrm{NH}$ & $(\mathrm{i}-\mathrm{Pr})_{2} \mathrm{NPh}$ & 15 & 90 & 6 \\
\hline 4 & $\mathrm{PhBr}$ & $\left(\mathrm{CH}_{3} \mathrm{CH}_{2} \mathrm{CH}_{2} \mathrm{CH}_{2}\right)_{2} \mathrm{NH}$ & $\left(\mathrm{CH}_{3} \mathrm{CH}_{2} \mathrm{CH}_{2} \mathrm{CH}_{2}\right)_{2} \mathrm{NPh}$ & 10 & 86 & 6 \\
\hline 5 & $\mathrm{PhBr}$ & $\left(\mathrm{CH}_{3}\right)_{2} \mathrm{CHCH}_{2} \mathrm{NH}$ & $\left(\mathrm{CH}_{3}\right)_{2} \mathrm{CHCH}_{2} \mathrm{NPh}$ & 10 & 80 & 12 \\
\hline 6 & $\mathrm{PhBr}$ & $(\mathrm{Cy})_{2} \mathrm{NH}$ & $(\mathrm{Cy})_{2} \mathrm{NPh}$ & 12 & 82 & 6 \\
\hline 7 & $\mathrm{PhBr}$ & 1-Phenylpiperazine & 1,4-Diphenylpiperazine & 10 & 87 & 13 \\
\hline 8 & $\mathrm{PhBr}$ & Piperidine & 1-Phenylpiperidine & 10 & 85 & 5 \\
\hline 9 & $\mathrm{PhBr}$ & Morpholine & 1-Phenylmorpholine & 8 & 95 & 6 \\
\hline 10 & $\mathrm{PhBr}$ & Indole & 1-Phenylindole & 18 & 76 & 6 \\
\hline 11 & $\mathrm{PhBr}$ & $\begin{array}{c}1,2,3,4- \\
\text { Tetrahydroquinoline }\end{array}$ & $\begin{array}{l}\text { 1-Phenyl-1,2,3,4- } \\
\text { tetrahydroquinoline }\end{array}$ & 14 & 86 & 6 \\
\hline 12 & $\mathrm{PhBr}$ & $\begin{array}{c}1,2,3,4- \\
\text { Tetrahydroisoquinoline }\end{array}$ & $\begin{array}{l}\text { 2-Phenyl-1,2,3,4- } \\
\text { tetrahydroisoquinoline }\end{array}$ & 12 & 91 & 14 \\
\hline 13 & $\mathrm{PhBr}$ & 1-Methylpiperazine & 1-Methyl-4-phenylpiperazine & 10 & 85 & 13 \\
\hline 14 & $\mathrm{PhBr}$ & Diphenylamine & Triphenylamine & 10 & 79 & 6 \\
\hline 15 & $\mathrm{PhCl}$ & $(\mathrm{i}-\mathrm{Pr})_{2} \mathrm{NH}$ & $(\mathrm{i}-\mathrm{Pr})_{2} \mathrm{NPh}$ & 15 & 87 & 6 \\
\hline 16 & $\mathrm{PhCl}$ & $\left(\mathrm{CH}_{3} \mathrm{CH}_{2} \mathrm{CH}_{2} \mathrm{CH}_{2}\right)_{2} \mathrm{NH}$ & $\left(\mathrm{CH}_{3} \mathrm{CH}_{2} \mathrm{CH}_{2} \mathrm{CH}_{2}\right)_{2} \mathrm{NPh}$ & 14 & 90 & 6 \\
\hline 17 & $\mathrm{PhCl}$ & Piperidine & 1-Phenylpiperidine & 12 & 82 & 5 \\
\hline 18 & $\mathrm{PhCl}$ & Morpholine & 1-Phenylmorpholine & 10 & 92 & 6 \\
\hline 19 & $\mathrm{PhCl}$ & Indole & 1-Phenylindole & 20 & 72 & 6 \\
\hline 20 & $\mathrm{PhCl}$ & Tetrahydroquinoline & 1-Phenyltetrahydroquinoline & 15 & 84 & 6 \\
\hline 21 & & morpholine & & 12 & 92 & 15 \\
\hline 22 & & morpholine & & 10 & 82 & 6 \\
\hline 23 & & morpholine & & 14 & 88 & 5 \\
\hline 24 & $\mathrm{PhBr}$ & $\left(\mathrm{CH}_{3} \mathrm{CH}_{2} \mathrm{CH}_{2} \mathrm{CH}_{2}\right)_{2} \mathrm{NH}$ & $\left(\mathrm{CH}_{3} \mathrm{CH}_{2} \mathrm{CH}_{2} \mathrm{CH}_{2}\right)_{2} \mathrm{NPh}$ & 16 & 80 & 6 \\
\hline 25 & $\mathrm{PhBr}$ & Piperidine & 1-Phenylpiperidine & 18 & 76 & 5 \\
\hline 26 & $\mathrm{PhBr}$ & Morpholine & 1-Phenylmorpholine & 16 & 89 & 6 \\
\hline
\end{tabular}

${ }^{a}$ Optimized conditions: $2.0 \mathrm{mmol}$ aryl halide, $3.0 \mathrm{mmol}$ amine, $3.0 \mathrm{mmol} \mathrm{KO}{ }^{t} \mathrm{Bu}$, and $5 \mathrm{~mol} \% \mathrm{TBAB}$ in $3 \mathrm{~mL}$ of $\mathrm{DMSO} .{ }^{b}$ Time in minutes. ${ }^{c}$ Isolated yield.

$2 \mathrm{H}), 6.64-6.61(\mathrm{~m}, 3 \mathrm{H}), 3.23-3.20(\mathrm{~m}, 4 \mathrm{H}), 1.62-1.57(\mathrm{~m}$, $4 \mathrm{H}), 0.93-0.89(\mathrm{~m}, 6 \mathrm{H}){ }^{5}$

$\mathrm{N}, \mathrm{N}$-Diisopropylaniline (3). $320 \mathrm{mg}, 90 \%$ yield, clear liquid; ${ }^{1} \mathrm{H}$ NMR $\left(\mathrm{CDCl}_{3}, 400 \mathrm{MHz}, \mathrm{ppm}\right): \delta 7.24-7.16(\mathrm{~m}, 2 \mathrm{H})$, 6.89-6.76 (m, 3H), 3.77-3.74 (m, 2H), 1.20-1.18 (d, $J=6.5$ $\mathrm{Hz}, 12 \mathrm{H})^{6}$

$N, N$-Dibutylaniline (4). $353 \mathrm{mg}, 86 \%$ yield, light yellow liquid; ${ }^{1} \mathrm{H}$ NMR $\left(\mathrm{CDCl}_{3}, 400 \mathrm{MHz}, \mathrm{ppm}\right): \delta 7.20-7.16(\mathrm{~m}$, $2 \mathrm{H}), 6.64-6.60(\mathrm{~m}, 3 \mathrm{H}), 3.26-3.22(\mathrm{t}, J=7.6 \mathrm{~Hz}, 4 \mathrm{H}), 1.57-$ $1.53(\mathrm{~m}, 4 \mathrm{H}), 1.35-1.31(\mathrm{~m}, 4 \mathrm{H}), 0.96-0.92(\mathrm{t}, J=7.6 \mathrm{~Hz}$, $6 \mathrm{H}){ }^{6}$

$\mathrm{N}, \mathrm{N}$-Diisobutylaniline (5). $328 \mathrm{mg}, 80 \%$ yield, clear to light yellow liquid; ${ }^{1} \mathrm{H} \mathrm{NMR}\left(\mathrm{CDCl}_{3}, 400 \mathrm{MHz}, \mathrm{ppm}\right): \delta 7.19-7.16$ $(\mathrm{m}, 2 \mathrm{H}), 6.64-6.59(\mathrm{~m}, 3 \mathrm{H}), 3.13-3.11(\mathrm{~d}, J=7.2 \mathrm{~Hz}, 4 \mathrm{H})$, $2.08-2.05(\mathrm{~m}, 2 \mathrm{H}), 0.89-0.87(\mathrm{~d}, J=6.4 \mathrm{~Hz}, 12 \mathrm{H}){ }^{12}$

$\mathrm{N}, \mathrm{N}$-Dicyclohexylaniline (6). $422 \mathrm{mg}, 82 \%$ yield, light yellow liquid; ${ }^{1} \mathrm{H} \mathrm{NMR}\left(\mathrm{CDCl}_{3}, 400 \mathrm{MHz}, \mathrm{ppm}\right): \delta 7.24-7.14$ $(\mathrm{m}, 2 \mathrm{H}), 6.93-6.78(\mathrm{~m}, 3 \mathrm{H}), 3.25-3.19(\mathrm{~m}, 2 \mathrm{H}), 1.75(\mathrm{~m}$, $8 \mathrm{H}), 1.63-1.48(\mathrm{~m}, 6 \mathrm{H}), 1.35-1.24(6 \mathrm{H}), 1.31-1.08(\mathrm{~m}$, $2 \mathrm{H}){ }^{6}$

1,4-Diphenylpiperazine (7). $414 \mathrm{mg}$, $87 \%$ yield, yellow to light brown solid, mp $159-160{ }^{\circ} \mathrm{C}$; ${ }^{1} \mathrm{H}$ NMR $\left(\mathrm{CDCl}_{3}, 400\right.$ $\mathrm{MHz}, \mathrm{ppm}): \delta 7.33-7.29(\mathrm{~m}, 4 \mathrm{H}), 7.01-6.99(\mathrm{~d}, J=7.6 \mathrm{~Hz}$, $4 \mathrm{H}), 6.92-6.89(\mathrm{t}, J=7.2 \mathrm{~Hz}, 2 \mathrm{H}), 3.35(\mathrm{~s}, 8 \mathrm{H}){ }^{13}$

1-Phenylpiperidine (8). $274 \mathrm{mg}, 85 \%$ yield, light yellow liquid; ${ }^{1} \mathrm{H}$ NMR $\left(\mathrm{CDCl}_{3}, 400 \mathrm{MHz}, \mathrm{ppm}\right): \delta 7.26-7.22(\mathrm{~m}$, 
$2 \mathrm{H}), 6.94-6.92(\mathrm{~m}, 2 \mathrm{H}), 6.83-6.81(\mathrm{~m}, 1 \mathrm{H}), 3.15-3.13(\mathrm{t}, J$ $=5.2 \mathrm{~Hz}, 4 \mathrm{H}), 1.71-1.69(\mathrm{~m}, 4 \mathrm{H}), 1.59-1.56(\mathrm{~m}, 2 \mathrm{H}) .^{5}$

1-Phenylmorpholine (9). $310 \mathrm{mg}, 95 \%$ yield, brown crystalline chunks, mp 51-53 ${ }^{\circ} \mathrm{C}$; ${ }^{1} \mathrm{H}$ NMR $\left(\mathrm{CDCl}_{3}, 400\right.$ $\mathrm{MHz}, \mathrm{ppm}) \delta 7.29-7.25(\mathrm{~m}, 2 \mathrm{H}), 6.92-6.87(\mathrm{~m}, 3 \mathrm{H}), 3.85(\mathrm{t}$, $J=4.7 \mathrm{~Hz}, 4 \mathrm{H}), 3.14(\mathrm{t}, J=5 \mathrm{~Hz}, 4 \mathrm{H}){ }^{6}$

1-Phenyl-1H-indole (10). $293 \mathrm{mg}, 76 \%$ yield, yellow to brown liquid; ${ }^{1} \mathrm{H} \mathrm{NMR}\left(\mathrm{CDCl}_{3}, 400 \mathrm{MHz}, \mathrm{ppm}\right): \delta 7.71-$ 7.7 .69 (d, $J=8 \mathrm{~Hz}, 1 \mathrm{H}), 7.59-7.56$ (d, $J=8.4 \mathrm{~Hz}, 1 \mathrm{H}), 7.52-$ $7.51(\mathrm{~d}, J=4 \mathrm{~Hz}, 4 \mathrm{H}), 7.36-7.34(\mathrm{~m}, 2 \mathrm{H}), 7.25-7.17(\mathrm{~m}$, $2 \mathrm{H}), 6.69-6.68(\mathrm{~d}, J=3.2 \mathrm{~Hz}, 1 \mathrm{H}){ }^{6}$

1-Phenyl-1,2,3,4-tetrahydroquinoline (11). $360 \mathrm{mg}, 86 \%$ yield, yellow to brown liquid; ${ }^{1} \mathrm{H}$ NMR $\left(\mathrm{CDCl}_{3}, 400 \mathrm{MHz}\right.$, ppm): $\delta 7.35-7.31(\mathrm{t}, J=7.8 \mathrm{~Hz}, 2 \mathrm{H}), 7.24-7.22(\mathrm{~d}, J=7.6$ $\mathrm{Hz}, 2 \mathrm{H}), 7.10-7.05(\mathrm{~m}, 2 \mathrm{H}), 6.92-6.90(\mathrm{t}, J=3.8 \mathrm{~Hz}, 1 \mathrm{H})$, $6.75-6.70(\mathrm{~m}, 2 \mathrm{H}), 3.64-3.61(\mathrm{t}, J=5.6 \mathrm{~Hz}, 2 \mathrm{H}), 2.86-2.83$ $(\mathrm{t}, J=6.4 \mathrm{~Hz}, 2 \mathrm{H}), 2.05-2.02$ (quint, $2 \mathrm{H}) .^{15}$

2-Phenyl-1,2,3,4-tetrahydroisoquinoline (12). $380 \mathrm{mg}$, $91 \%$ yield, pale orange solid; ${ }^{1} \mathrm{H}$ NMR $\left(\mathrm{CDCl}_{3}, 400 \mathrm{MHz}\right.$, ppm): $\delta 7.32-7.24(\mathrm{~m}, 2 \mathrm{H}), 7.20-7.18(\mathrm{~m}, 4 \mathrm{H}), 7.01-6.99$ $(\mathrm{m}, 2 \mathrm{H}), 6.86-6.84(\mathrm{~m}, 1 \mathrm{H}), 4.44-4.42(\mathrm{~m}, 2 \mathrm{H}), 3.59-3.56$ (m, 2H), 3.01-2.98 (m, 2H). ${ }^{13}$

1-Methyl-4-phenylpiperazine (13). $299 \mathrm{mg}, 85 \%$ yield, clear liquid; ${ }^{1} \mathrm{H}$ NMR $\left(\mathrm{CDCl}_{3}, 400 \mathrm{MHz}, \mathrm{ppm}\right): \delta$ 7.25-7.23 (m, $2 \mathrm{H}), 6.93-6.91(\mathrm{~d}, J=8 \mathrm{~Hz}, 3 \mathrm{H}), 3.21-3.18(\mathrm{t}, J=5 \mathrm{~Hz}$, $4 \mathrm{H}), 2.57-2.55(\mathrm{t}, J=5 \mathrm{~Hz}, 4 \mathrm{H}), 2.33(\mathrm{~s}, 3 \mathrm{H}){ }^{13}$

Triphenylamine (14). $387 \mathrm{mg}, 79 \%$ yield, off-white crystalline solid, mp $125-127{ }^{\circ} \mathrm{C} ;{ }^{1} \mathrm{H}$ NMR $\left(\mathrm{CDCl}_{3}, 400\right.$ $\mathrm{MHz}, \mathrm{ppm}): \delta 7.32-7.28(\mathrm{~m}, 6 \mathrm{H}), 7.17-7.15(\mathrm{~m}, 6 \mathrm{H}), 7.08-$ $7.04(\mathrm{~m}, 4 \mathrm{H}){ }^{6}$

4-(4-Fluorophenyl)morpholine and 4-(3-Fluorophenyl)morpholine (21). $333 \mathrm{mg}$, 92\% yield, colorless liquid; Pisomer ${ }^{1} \mathrm{H} \mathrm{NMR}\left(\mathrm{CDCl}_{3}, 400 \mathrm{MHz}, \mathrm{ppm}\right): \delta 6.98-6.94(\mathrm{t}, J=$ $8.8 \mathrm{~Hz}, 1 \mathrm{H}), 6.86-6.83(\mathrm{dd}, J=4.8 \mathrm{~Hz}, 1 \mathrm{H}), 3.85-3.84(\mathrm{t}, J=$ $7.6 \mathrm{~Hz}, 2 \mathrm{H}), 3.07-3.05(\mathrm{t}, J=5 \mathrm{~Hz}, 2 \mathrm{H})$. m-isomer ${ }^{1} \mathrm{H}$ NMR $\left(\mathrm{CDCl}_{3}, 400 \mathrm{MHz}, \mathrm{ppm}\right): \delta 7.19(\mathrm{dd}, 0.5 \mathrm{H}), 6.64(\mathrm{dd}, 0.5 \mathrm{H})$, 6.55-6.54 (m, $1 \mathrm{H}), 3.83-3.82(\mathrm{t}, J=5 \mathrm{~Hz}, 2 \mathrm{H}), 3.15-3.13(\mathrm{t}$, $J=4.8 \mathrm{~Hz}, 2 \mathrm{H}){ }^{14}$ A $1: 1$ ratio was obtained; therefore, integration values should be multiplied by 2 to obtain the reported spectra in the literature of each individual isomer. ${ }^{15,16}$

4-(4-Methylphenyl)morpholine and 4-(3-Methylphenyl)morpholine (22). $290 \mathrm{mg}$, yellow to brown solid, $82 \%$ yield. Pisomer ${ }^{1} \mathrm{H} \mathrm{NMR}\left(\mathrm{CDCl}_{3}, 400 \mathrm{MHz}, \mathrm{ppm}\right): \delta 7.08-7.06$ (d, $J$ $=8 \mathrm{~Hz}, 1 \mathrm{H}), 6.83-6.81(\mathrm{~d}, J=8.8 \mathrm{~Hz}, 1 \mathrm{H}), 3.86-3.83(\mathrm{t}, J=$ $4.6 \mathrm{~Hz}, 2 \mathrm{H}), 3.10-3.08(\mathrm{t}, J=5 \mathrm{~Hz}, 2 \mathrm{H}), 2.28(\mathrm{~s}, 1.5 \mathrm{H}) . \mathrm{m}-$ isomer ${ }^{1} \mathrm{H}$ NMR $\left(\mathrm{CDCl}_{3}, 400 \mathrm{MHz}, \mathrm{ppm}\right): \delta 7.15(\mathrm{t}, J=4.4$ $\mathrm{Hz}, 0.5 \mathrm{H}), 6.73-6.71(\mathrm{~m}, 1.5 \mathrm{H}), 3.86-3.84(\mathrm{t}, J=4.6 \mathrm{~Hz}$, $2 \mathrm{H}), 3.14-3.12(\mathrm{t}, J=4.8 \mathrm{~Hz}, 2 \mathrm{H}), 2.31(\mathrm{~s}, 1.5 \mathrm{H})$. A $1: 1$ ratio was obtained; therefore, integration values should be multiplied by 2 to obtain the reported spectra in the literature of each individual isomer. ${ }^{6}$

4-(4-Methoxyphenyl)morpholine and 4-(3Methoxyphenyl)morpholine (23). $340 \mathrm{mg}$, clear liquid, 88\% yield. P-isomer ${ }^{1} \mathrm{H}$ NMR $\left(\mathrm{CDCl}_{3}, 400 \mathrm{MHz}, \mathrm{ppm}\right): \delta 6.89$ (d, $J=9.6 \mathrm{~Hz}, 2 \mathrm{H}), 6.85-6.83(\mathrm{~d}, J=8 \mathrm{~Hz}, 1 \mathrm{H}), 3.86-3.84(\mathrm{t}, J=$ $6.4 \mathrm{~Hz}, 2 \mathrm{H}), 3.78(\mathrm{~s}, 1.5 \mathrm{H}), 3.05-3.03(\mathrm{t}, J=4.6 \mathrm{~Hz}, 2 \mathrm{H}) . \mathrm{m}-$ isomer ${ }^{1} \mathrm{H}$ NMR $\left(\mathrm{CDCl}_{3}, 400 \mathrm{MHz}, \mathrm{ppm}\right): \delta 7.19-7.15(\mathrm{~m}$, $0.5 \mathrm{H}), 6.53-6.51(\mathrm{~d}, J=8 \mathrm{~Hz}, 0.5 \mathrm{H}), 6.44(\mathrm{~s}, 0.5 \mathrm{H}), 3.82-$ $3.80(\mathrm{t}, J=6.4 \mathrm{~Hz}, 2 \mathrm{H}), 3.76(\mathrm{~s}, 1.5 \mathrm{H}), 3.15-3.13(\mathrm{t}, J=4.6$ $\mathrm{Hz}, 2 \mathrm{H})$. A 1:1 ratio was obtained; therefore, integration values should be multiplied by 2 to obtain the reported spectra in the literature of each individual isomer. ${ }^{5}$

\section{ASSOCIATED CONTENT}

\section{Supporting Information}

The Supporting Information is available free of charge on the ACS Publications website at DOI: 10.1021/acsomega.8b01426.

Copies of ${ }^{1} \mathrm{H}$ NMR spectra of all compounds (PDF)

\section{AUTHOR INFORMATION}

\section{Corresponding Author}

*E-mail: Coop-acoop@rx.umaryland.edu (A.C.).

ORCID

Andrew Coop: 0000-0002-4913-9447

Author Contributions

${ }^{\dagger}$ C.R.J. and M.I.A. contributed equally.

Notes

The authors declare no competing financial interest.

\section{ACKNOWLEDGMENTS}

This work was funded by NIMH (R01 107499-01A1).

\section{REFERENCES}

(1) (a) Fischer, C.; Koenig, B. Palladium- and copper-mediatedNaryl bond formation reactions for the synthesis of biological active compounds. Beilstein J. Org. Chem. 2011, 7, 59-74. (b) Muci, A. R.; Buchwald, S. L. Practical Palladium Catalysts for C-N and C-O Bond Formation. Top. Curr. Chem. 2002, 219, 131-209. (c) Ruiz-Castillo, P.; Buchwald, S. L. Applications of Palladium-Catalyzed C-N CrossCoupling Reactions. Chem. Rev. 2016, 116, 12564-12649. (d) Ahneman, D. T.; Estrada, J. G.; Lin, S.; Dreher, S. D.; Doyle, A. G. Predicting reaction performance in $\mathrm{C}-\mathrm{N}$ cross-coupling using machine learning. Science 2018, 360, 186. Badigenchala, S.; Sekar, G. NIS Mediated Cross-Coupling of $\mathrm{C}\left(\mathrm{sp}^{2}\right)-\mathrm{H}$ and N-H Bonds: A Transition-Metal-Free Approach toward Indolo[1,2-a]quinazolinones. J. Org. Chem. 2017, 82, 7657-7665.

(2) For the metal catalyzed reactions, see: (a) Kwong, F. Y.; Buchwald, S. L. Mild and Efficient Copper-Catalyzed Amination of Aryl Bromides with Primary Alkylamines. Org. Lett. 2003, 5, 793796. (b) Okano, K.; Tokuyama, H.; Fukuyama, T. Synthesis of Secondary Arylamines through Copper-Mediated Intermolecular Aryl Amination. Org. Lett. 2003, 5, 4987-4990. (c) Antilla, J. C.; Baskin, J. M.; Barder, T. E.; Buchwald, S. L. Copper-Diamine-CatalyzedNArylation of Pyrroles, Pyrazoles, Indazoles, Imidazoles, and Triazoles. J. Org. Chem. 2004, 69, 5578-5587. (d) Hu, T.; Li, C. Synthesis of Lactams via Copper-Catalyzed Intramolecular Vinylation of Amides. Org. Lett. 2005, 7, 2035-2038. (e) Rao, H.; Fu, H.; Jiang, Y.; Zhao, Y. Copper-Catalyzed Arylation of Amines Using Diphenyl Pyrrolidine-2phosphonate as the New Ligand. J. Org. Chem. 2005, 70, 8107-8109. (f) Zim, D.; Buchwald, S. L. An Air and Thermally Stable OneComponent Catalyst for the Amination of Aryl Chlorides. Org. Lett. 2003, 5, 2413-2415. (g) Urgaonkar, S.; Nagarajan, M.; Verkade, J. G. $\mathrm{P}\left(\mathrm{i}-\mathrm{BuNCH}_{2} \mathrm{CH}_{2}\right)_{3} \mathrm{~N}$ : An Effective Ligand in the PalladiumCatalyzed Amination of Aryl Bromides and Iodides. J. Org. Chem. 2003, 68, 452-459. (h) Shen, Q.; Ogata, T.; Hartwig, J. F. Highly Reactive, General and Long-Lived Catalysts for Palladium-Catalyzed Amination of Heteroaryl and Aryl Chlorides, Bromides, and Iodides: Scope and Structure-Activity Relationships. J. Am. Chem. Soc. 2008, 130, 6586-6596. (i) Zhou, W.; Fan, M.; Yin, J.; Jiang, Y.; Ma, D. CuI/Oxalic Diamide Catalyzed Coupling Reaction of (Hetero)Aryl Chlorides and Amines. J. Am. Chem. Soc. 2015, 137, 11942-11945. (j) Zhang, Y.; Yang, X.; Yao, Q.; Ma, D. CuI/DMPAO-Catalyzed NArylation of Acyclic Secondary Amines. Org. Lett. 2012, 14, 30563059. (k) Kurandina, D. V.; Eliseenkov, E. V.; Khaibulova, T. S.; Petrov, A. A.; Boyarskiy, V. P. Copper-catalyzed C-N bond crosscoupling of aryl halides and amines in water in the presence of ligand derived from oxalyl dihydrazide: scope and limitation. Tetrahedron 
2015, 71, 7931-7937. (1) Sharghi, H.; Sepehri, S.; Aberi, M. Cu(II) complex of pyridine-based polydentate as a novel, efficient, and highly reusable catalyst in C-N bond-forming reaction. Mol. Divers. 2017, 21, 855-864. (m) Zhao, X.; She, Y.; Fang, K.; Li, G. CuCl-Catalyzed Ullmann-Type C-N Cross-Coupling Reaction of Carbazoles and 2Bromopyridine Derivatives. J. Org. Chem. 2017, 82, 1024-1033. (n) Pradhan, S.; De, P. B.; Punniyamurthy, T. Copper(II)-Mediated Chelation-Assisted Regioselective N-Naphthylation of Indoles, Pyrazoles and Pyrrole through Dehydrogenative Cross-Coupling. J. Org. Chem. 2017, 82, 4883-4890. (o) Tian, X.; Lin, J.; Zou, S.; Lv, J.; Huang, Q.; Zhu, J.; Huang, S.; Wang, Q. [Pd( $(\operatorname{Pr} * R)($ acac $) C l]$ : Efficient bulky Pd-NHC catalyst for Buchwald-Hartwig C-N crosscoupling reaction. J. Organomet. Chem. 2018, 861, 125-130.

(3) (a) Samblanet, D. C.; Schmidt, J. A. R. Efficient catalytic aryl amination of bromoarenes using 3-iminophosphine palladium(II) chloride. J. Organomet. Chem. 2012, 720, 7-18. (b) Bolliger, J. L.; Frech, C. M. Transition metal-free amination of aryl halides-A simple and reliable method for the efficient and high-yielding synthesis of $\mathrm{N}$ arylated amines. Tetrahedron 2009, 65, 1180-1187. (c) Sun, C.-L.; Shi, Z.-J. Transition-Metal-Free Coupling Reactions. Chem. Rev. 2014, 114, 9219-9280.

(4) Lu, J.-M.; Huang, P.; He, B.-Y.; Wang, H.-M. Transition-MetalFree, Potassium tert-Butoxide/Dimethyl Sulfoxide Mediated Amination between Tertiary Amines and Aryl Halides. Synthesis 2015, 47, 221-227.

(5) Fang, Y.; Zheng, Y.; Wang, Z. Direct Base-Assisted C-N Bond Formation between Aryl Halides and Aliphatic Tertiary Amines under Transition-Metal-Free Conditions. Eur. J. Org. Chem. 2012, 14951498.

(6) Shi, L.; Wang, M.; Fan, C.-A.; Zhang, F.-M.; Tu, Y.-Q. Rapid and Efficient Microwave-Assisted Amination of Electron-Rich Aryl Halides without a Transition-Metal Catalyst. Org. Lett. 2003, 5, 3515-3517.

(7) Johnson, C. R.; Lavergne, O. M. Alkylation of sulfoximine and related compounds at the imino nitrogen under phase-transfer conditions. J. Org. Chem. 1993, 58, 1922-1923.

(8) Wang, C.; Hang, T.; Zhang, H. Microwave-PromotedNAlkylation of Acridones Without Solvent. Synth. Commun. 2003, 33, 451-456.

(9) Majumdar, K. C.; Sarkar, S.; Ghosh, S. Studies in Thio-Claisen Rearrangement: Regioselective Synthesis of Thiopyrano[2,3-b]pyran2-ones and Thieno[2,3-b]pyran-2-ones. Synth. Commun. 2004, 34, $1265-1275$.

(10) Lebel, H.; Morin, S.; Paquet, V. Alkylation of Phosphine Boranes by Phase-Transfer Catalysis. Org. Lett. 2003, 5, 2347-2349. (11) (a) Li, J.-H.; Li, J.-L.; Xie, Y.-X. TBAB-Promoted Ligand-Free Copper-Catalyzed Cross-Coupling Reactions of Aryl Halides with Arylboronic Acids. Synthesis 2007, 984-988. (b) Tang, B.-X.; Wang, F.; Li, J.-H.; Xie, Y.-X.; Zhang, M.-B. Reusable $\mathrm{Cu}_{2} \mathrm{O} / \mathrm{PPh}_{3} / \mathrm{TBAB}$ System for the Cross-Couplings of Aryl Halides and Heteroaryl Halides with Terminal Alkynes. J. Org. Chem. 2007, 72, 6294-6297.

(12) Gurrapu, S.; Jonnalagadda, S. K.; Alam, M. A.; Nelson, G. L.; Sneve, M. G.; Drewes, L. R.; Mereddy, V. R. Monocarboxylate Transporter 1 Inhibitors as Potential Anticancer Agents. ACS Med. Chem. Lett. 2015, 6, 558-561.

(13) Lin, Y.; Li, M.; Ji, X.; Wu, J.; Cao, S. n-Butyllithium-mediated synthesis of $\mathrm{N}$-aryl tertiary amines by reactions of fluoroarenes with secondary amines at room temperature. Tetrahedron 2017, 73, 14661472.

(14) Oss, G.; de Vos, S. D.; Luc, K. N. H.; Harper, J. B.; Nguyen, T. V. Tropylium-Promoted Oxidative Functionalization of Tetrahydroisoquinolines. J. Org. Chem. 2018, 83, 1000-1010.

(15) Zhang, Z.-M.; Gao, Y.-J.; Lu, J.-M. Synthesis of N -heterocyclic carbene-Pd(II) complexes and their catalytic activity in the BuchwaldHartwig amination of aryl chlorides. Tetrahedron 2017, 73, 73087314.

(16) Heaney, H. The Benzyne and Related Intermediates. Chem. Rev. 1962, 62, 81-97. 